\title{
A Note on Primitive Permutation Groups of Prime Power Degree
}

\author{
Qian Cai ${ }^{1}$ and Hua Zhang ${ }^{2}$ \\ ${ }^{1}$ Department of Mathematics, Puer University, Puer 665000, China \\ ${ }^{2}$ School of Mathematics, Yunnan Normal University, Kunming 650091, China \\ Correspondence should be addressed to Hua Zhang; zhdahua@gmail.com
}

Received 7 October 2014; Accepted 23 December 2014

Academic Editor: Hong J. Lai

Copyright (c) 2015 Q. Cai and H. Zhang. This is an open access article distributed under the Creative Commons Attribution License, which permits unrestricted use, distribution, and reproduction in any medium, provided the original work is properly cited.

Primitive permutation groups of prime power degree are known to be affine type, almost simple type, and product action type. At the present stage finding an explicit classification of primitive groups of affine type seems untractable, while the product action type can usually be reduced to almost simple type. In this paper, we present a short survey of the development of primitive groups of prime power degree, together with a brief description on such groups.

\section{Introduction}

Transitive groups, in particular, primitive groups, of special degrees have received much attention in the literature. As early as 1832, Galois showed that the projective linear groups $\mathrm{PSL}_{2}(p)$ have permutation representations of degree $p$ with $p=5,7$, and 11. In 1861, Mathieu discovered his famous multiply transitive groups, including two sporadic simple groups $M_{11}$ and $M_{23}$, of degrees 11 and 23, respectively. In 1901, Burnside [1] classified transitive groups of prime degree, showing that such groups are doubly transitive or contain a normal regular $p$-group with $p$ prime. As a well-known consequence of the classification of the finite simple groups (CFSG), all doubly transitive groups are known [2, Theorem 5.3], and hence all doubly transitive groups of prime degree are known. It means that, by using the CFSG, one can easily obtain Burnside's classification result. Later in 1983 Guralnick [3] studied primitive simple groups of prime power degree, in 1985 Liebeck and Saxl [4] classified primitive groups of odd degree, and in $2003 \mathrm{Li}$ and Seress classified primitive groups of square-free degree [5]. In [6], Li gave a list of primitive permutation groups of degree $n$, where $n=2 \cdot 3^{r}, 5 \cdot 3^{r}$, or $10 \cdot 3^{r}$, with $r$ a prime.

One of the pioneers of investigating primitive permutation groups of prime power degree is G. Jones in 1975, in his Ph.D. thesis. At that time, an explicit description on such groups was not available because the classification of the finite simple groups had not been completed. In 1976, Praeger also studied primitive permutation groups of prime power degree [7]. Then, in 1979 at the Santa Cruz Conference in finite groups, M. O’Nan and L. L. Scott independently proposed a classification scheme for finite primitive groups, which became a theorem of O'Nan and Scott and finally the O'Nan-Scott Theorem; see [8]. This theorem has been proved to be very important in studying finite primitive groups. Nevertheless, when dealing with primitive groups of special degrees, more work needs to be done.

In 2002, Dobson and Witte [9] determined all the transitive groups of degree $p^{2}$, with $p$ a prime, whose Sylow $p$-subgroup is not isomorphic to the wreath product $\mathbb{Z}_{p}$ ? $\mathbb{Z}_{p}$. Hence, it is a natural next step to carry out a study on transitive groups of degree $p^{3}$ or, more generally, of degree $p^{k}$, with $p$ a prime.

In recent years, the problem of determining transitive (or primitive) permutation groups of special degree is closely related to some important combinatorial problems, such as the problem of classifying symmetric graphs, symmetric Cayley graphs, edge-transitive graphs, and half-arc-transitive graphs, of specific degree.

In this paper, we take a simple retrospect on the analyzing process of the primitive permutation groups of prime power 
degree and present a brief description of primitive permutation groups of degree $p^{r}$, with $p$ a prime. In the spirit of the Burnside Theorem and Dobson's work, here we may assume that $r \geq 3$. This work forms the first part of a larger program, namely, to classify transitive permutation groups of prime power degree.

Our description of primitive permutation groups of prime power degree is given in the following theorem.

Theorem 1. Let $G$ be a primitive permutation group of degree $p^{r}$, with $p$ primes, where $r \geq 3$. Then, one of the following holds.

(a) $G$ is doubly transitive and either

(i) $G$ is affine type, $G=\mathbb{Z}_{p}^{r} \rtimes G_{\alpha} \leq A \Gamma L_{r}(p)$, and $G_{\alpha}$ is transitive on the nonzero vectors of $\mathbb{Z}_{p}^{r}$, or

(ii) $G$ is almost simple type and $G$ is one of the following groups: $A_{p^{r}}, S_{p^{r}}$, and $P S L_{n}(q) \leq G \leq$ $P \Gamma L_{n}(q)$, where $\left(q^{n}-1\right) /(q-1)=p^{r}$.

(b) $G$ is simply primitive, and one of the following is true:

(i) $G$ is affine type, $G=\mathbb{Z}_{p}^{r}: G_{\alpha} \leq A \Gamma L_{r}(p)$, and $G_{\alpha}$ is irreducible but not transitive on the nonzero vectors of $\mathbb{Z}_{p}^{r}$;

(ii) $G$ is almost simple type, and $P S U_{4}(2) \cong$ $P S p_{4}(3) \leq G \leq P S U_{4}(2) .2 \cong P S p_{4}(3) .2$, with $p=r=3$;

(iii) $G$ is product action type, and $T^{d}=\operatorname{soc}(G) \triangleleft$ $G \leq U<K$, where $1<d \leq r, d \mid r$, and $K$ is a transitive subgroup of $S_{d} ; \operatorname{soc}(U)=T \leq S_{p^{r / d}}$ lies in the following list: $A_{p^{r / d}} ; P S L_{n}(q)$ with $\left(q^{n}-\right.$ $1) /(q-1)=p^{r / d} ; M_{11}, P_{2}(11)$ with $p=11$, $r=d ; M_{23}$ with $p=23, r=d ; P_{4}(2)$ with $1<d=r / 3$.

Remark 2. The subgroups of $\mathrm{GL}_{n}(q)$ which are transitive on the nonzero vectors of $\mathbb{Z}_{q}^{n}$ are determined by Hering in [10].

\section{Primitive Groups of Degree $p^{r}$}

Let $\Omega$ be a nonempty set. Recall that the symmetric group $\operatorname{Sym}(\Omega)$ is defined to be the group of all permutations of $\Omega$. A permutation group $G$ on $\Omega$ is simply a subgroup of the symmetric group $\operatorname{Sym}(\Omega)$, and the size $|\Omega|$ is called the degree of $G$. If $|\Omega|=n$, then $\operatorname{Sym}(\Omega)$ is also denoted by $S_{n}$. The set of the even permutations of $S_{n}$ forms a subgroup of $S_{n}$, which is called the alternating group of degree $n$ and is denoted by $\operatorname{Alt}(\Omega)$ or $A_{n}$ if $|\Omega|=n$.

Let $G \leq \operatorname{Sym}(\Omega)$. For $\alpha \in \Omega$, we use $G_{\alpha}$ to denote the point stabilizer of $G$ with respect to $\alpha$. The orbit of $G$ containing $\alpha$ is defined to be the set $\alpha^{G}:=\left\{\alpha^{g} \mid g \in G\right\}$. It is well known that $\left|\alpha^{G}\right|=|G| /\left|G_{\alpha}\right|$. The group $G$ is called transitive if, for any two points $\alpha, \beta \in \Omega$, there exists an element $g \in G$ such that $\alpha^{\mathscr{g}}=\beta$, which is equivalent to saying that $G$ has only one orbit on $\Omega$. A group $G \leq \operatorname{Sym}(\Omega)$ is called semiregular if $G_{\alpha}=1$ for every $\alpha \in \Omega$, and $G$ is called regular if $G$ is transitive and semiregular. Observe that any proper subgroup of a regular group is semiregular but not regular.

For a set $\Omega$, let $\mathscr{B}=\left\{B_{1}, B_{2}, \ldots, B_{m}\right\}$ be a set of subsets of $\Omega$. Then, $\mathscr{B}$ is said to be a partition of $\Omega$ if $\Omega$ is the disjoint union of $B_{i}$; that is, $\Omega=B_{1} \cup B_{2} \cdots \cup B_{m}$, and $B_{i} \cap B_{j}=$ $\emptyset$ for $i \neq j$. There are two trivial partitions of $\Omega: \mathscr{B}=\{\Omega\}$ and $\mathscr{B}=\left\{B_{1}, B_{2}, \ldots, B_{m}\right\}$ with $\left|B_{i}\right|=1$. Let $G$ be a transitive permutation group on $\Omega$. A partition $\mathscr{B}$ of $\Omega$ is said to be $G$ invariant, if, for any $g \in G$ and $B \in \mathscr{B}, B^{g} \in \mathscr{B}$. In this case, $\mathscr{B}$ is also said to be an imprimitive partition of $G$ on $\Omega$.

Let $G \leq \operatorname{Sym}(\Omega)$ be transitive. Then, $G$ is called primitive if it has no nontrivial $G$-invariant partition. If $\mathscr{B}$ is an imprimitive partition of $G$ on $\Omega$, then $|\mathscr{B}|$ divides $|\Omega|$, and so each transitive permutation group of prime degree is primitive. Let $\Omega^{(k)}$ be the set of all $k$-tuples of points in $\Omega$; that is,

$$
\Omega^{(k)}:=\left\{\left(\alpha_{1}, \alpha_{2}, \ldots, \alpha_{k}\right) \mid \alpha_{i} \in \Omega\right\}
$$

Then, one can define an action of $G$ on $\Omega^{(k)}$ by

$$
\left(\alpha_{1}, \alpha_{2}, \ldots, \alpha_{k}\right)^{g}=\left(\alpha_{1}^{g}, \alpha_{2}^{g}, \ldots, \alpha_{k}^{g}\right) .
$$

A group $G \leq \operatorname{Sym}(\Omega)$ is said to be $k$-transitive if $G$ is transitive on $\Omega^{(k)}$. A 2-transitive group is also called doubly transitive. It is well-known that a doubly transitive group is primitive. If $G$ is primitive but not doubly transitive, then we say that $G$ is simply primitive.

The primitivity of a transitive permutation group may be characterized by the maximality of its point stabilizer, that is, the following well-known result.

Proposition 3. A transitive permutation group $G \leq \operatorname{Sym}(\Omega)$, where $|\Omega| \geq 2$, is primitive if and only if each of its point stabilizers is a maximal subgroup of $G$.

The structure of finite primitive groups is characterized by the famous O'Nan-Scott Theorem (see [11, page 106] or [8]). By the O'Nan-Scott Theorem, finite primitive groups can be divided into five disjoint types, known as HA, AS, SD, PA, and TW. Thus, let $G \leq \operatorname{Sym}(\Omega)$ be a primitive permutation group, and let $H=\operatorname{soc}(G)$ be the socle of $G$, that is, the product of all minimal normal subgroups of $G$. Then, we can give a brief description of the five types of finite primitive groups as follows.

HA (holomorph affine): $H$ is a regular elementary abelian $p$-group $\mathbb{Z}_{p}^{m}$ for some prime $p,|\Omega|=p^{m}$, and $G=\mathbb{Z}_{p}^{m}: G_{\alpha} \leq \mathrm{A}^{2} \mathrm{~L}_{m}(p)$, where $G_{\alpha}$ is irreducible on $\mathbb{Z}_{p}^{m}$.

AS (almost simple): $H=T$ is a nonabelian simple group, and $T \unlhd G \leq \operatorname{Aut}(T)$.

SD (simple diagonal): $H \cong T^{m}$, where $m \geq 2,|\Omega|=$ $|T|^{m-1}$, and $H_{\alpha} \cong T$.

PA (product action): $H \cong T^{m}$, where $m \geq 2, d<$ $m, d \mid m$, and let $U$ be a primitive group of $l$ with $\operatorname{soc}(U) \cong T^{d}$; then, $G$ is isomorphic to a subgroup of $U_{2} \operatorname{Sym}(m / d)$, with the product action, and $|\Omega|=l^{m / d}$. 
TW (twisted wreath product): $H \cong T^{m}$, where $m \geq 6$, and $H$ is regular on $\Omega$, with $|\Omega|=|T|^{m}$.

Remark 4. In the above description, $T$ is a nonabelian simple group and $U$ is primitive of type AS or SD.

Lemma 5. Let $G$ be a primitive permutation group acting on a set $\Omega$, where $|\Omega|=p^{n}(n \in \mathbb{N})$ for some prime $p$. Then, the type of $G$ is $H A, A S$, or $P A$.

Proof. Let $G$ be a primitive permutation group acting on a set $\Omega$, let $H$ be the socle of $G$, and let $\alpha \in \Omega$. Then, by the O'Nan-Scott Theorem, the type of $G$ is HA, AS, SD, PA, or TW. Suppose that the type of $G$ is SD. Then, $m \geq 2,|\Omega|=$ $|T|^{m-1}=p^{a}$. Since $T$ is a nonabelian simple, this is not possible. Similarly the type of $G$ is not TW. Thus, the type of $G$ is HA, AS, or PA.

It is clear that if $n=1$, then the type of $G$ is HA or AS.

In [3], Guralnick classified finite simple group with a subgroup index a prime power.

Theorem 6 (Guralnick, [3]). Let $G$ be a nonabelian simple group, let $H<G$, and let $|[G: H]|=p^{a}$, where $a \in \mathbb{N}$ and $p$ is a prime. Then, one of the following holds.

(a) $G=A_{n}$ and $H \cong A_{n-1}$, with $n=p^{a}$.

(b) $G=P S L_{n}(q), H$ is the stabilizer of a line or a hyperplane, and $|[G: H]|=\left(q^{n}-1\right) /(q-1)=p^{a}$.

(c) $G=P S L_{2}(11)$ and $H \cong A_{5}$.

(d) $G=M_{23}$ and $H \cong M_{22}$ or $G=M_{11}$ and $H \cong M_{10}$.

(e) $G=P U_{4}(2) \cong P S p_{4}(3)$ and $H \cong \mathbb{Z}_{2}^{4} \rtimes A_{5}$.

As a result, we have the following.

Corollary 7. Let $G$ be a nonabelian simple group acting transitively on a set $\Omega$ with $|\Omega|=p^{a}$. Then, either $G$ acts doubly transitive on $\Omega$ or $G \cong P S U_{4}(2)$ acts simply primitively on $\Omega$, with $|\Omega|=27$.

Proof. Let $G$ act transitively on $\Omega$, where $|\Omega|=p^{a}$. Then, $[G$ : $\left.G_{\alpha}\right]=p^{a}$. Therefore, the pair $\left(G, G_{\alpha}\right)=(G, H)$ lies in the list of Theorem 6. Combining this list with the list of doubly transitive groups given in [2, Table on page 8], we conclude that $G$ acts doubly transitive on $\Omega$ unless $G \cong \operatorname{PSU}_{4}(2)$, which has a maximal subgroup $H \cong \mathbb{Z}_{2}^{4}: A_{5}$, of index 27 , and $G$ is simply primitive on $\Omega=[G: H]$. Therefore, the result is true.

We point out that if $p=2$, then either $G=A_{2^{a}}$ or $G=$ $\operatorname{PSL}_{2}(q)$, such that $q=2^{a}-1$ is a Mersenne prime.

In [12], Burnside proved the following result.

Theorem 8. The socle of a finite doubly transitive group is either a regular elementary abelian p-group or a nonabelian simple group.

It follows from the above result that a doubly transitive group is of type HA or AS.
Let $G$ be a doubly transitive group of degree $p^{n}$, with $p$ a prime. If the type of $G$ is HA, then the socle of $G$ is abelian, which is isomorphic to an elementary abelian group $\mathbb{Z}_{p}^{n}$. In this case, $G=\mathbb{Z}_{p}^{n}: G_{\alpha} \leq \operatorname{A\Gamma L}_{n}(p)$, and $G_{\alpha}$ is an irreducible subgroup of $\Gamma \mathrm{L}_{n}(p)$ which is transitive on nonzero vectors of $\mathbb{Z}_{p}^{n}$. Such groups $G$ have been determined by Huppert in [13] for soluble case and by Hering [10] for insoluble case.

If $G$ is simply primitive of affine type, then the problem is very hard, and there is a long standing open problem.

Open Problem 1. Find out all irreducible subgroups of $\mathrm{GL}_{n}(p)$ which are not transitive on the nonzero vectors of $\mathbb{Z}_{p}^{n}$.

If the type of $G$ is AS, then $G$ can be easily read off from Theorem 6. Note that doubly transitive groups with nonabelian socles are also listed in [2, page 8$]$, as a result of the classification of finite simple groups. By Theorem 6 or by inspecting the list, we see that the only doubly transitive groups of degree $p^{n}(n \geq 2)$ are as follows.

Theorem 9. Let $G$ be a doubly transitive group of degree $p^{n}$ $(n \geq 2)$ with nonabelian socle. Then, either $G=A_{p^{n}}$ or $G=S_{p^{n}}$ or $P S L_{k}(q) \leq G \leq P \Gamma L_{k}(q)$, where $\left(q^{k}-1\right) /(q-1)=p^{n}$.

Transitive groups of prime degree are known for a very long time and are given as follows.

Theorem 10 (see [11, page 99]). Let $G$ be a transitive group of prime $p$ degree. Then, one of the following statements holds.

(1) $G$ is primitive of holomorph affine type, and $\mathbb{Z}_{p} \leq G \leq$ $A G L_{1}(p)$.

(2) $G$ is primitive of almost simple type, and one of the following holds:

(i) $G=A_{p}$ or $S_{p}$;

(ii) $P S L_{n}(q) \leq G \leq P \Gamma L_{n}(q)$, where $\left(q^{n}-1\right) /(q-1)=$ $p$

(iii) $G=P S L_{2}(11)$ or $P \Gamma L_{2}(11), p=11$;

(iv) $G=M_{11}$ or $M_{23}$ and $p=11$ or 13 .

Primitive groups of large prime power degrees sometimes appear as the wreath product of primitive groups of small prime power degrees, in the product action. The spirit is the following well-known result.

Proposition 11 (see [11, page 50]). Suppose that $H$ and $K$ are nontrivial permutation groups acting on the sets $\Gamma$ and $\Delta$, respectively. Then, the wreath product $G=K 2 H$ is primitive in the product action on $\Omega=\Delta^{|\Gamma|}$ if and only if

(i) $K$ acts primitively but not regularly on $\Delta$;

(ii) $\Gamma$ is finite and $H$ acts transitively on $\Gamma$.

It follows from the above result that, for $|\Omega|=p^{n}$, with $p$ prime, we have the next corollary.

Corollary 12. The wreath product $G=K 2 H$ is primitive in the product action on $\Omega$ with $|\Omega|=p^{n}$, if and only if one of the following holds. 
(a) $n$ is a prime, $K$ is one as listed in (2) of Theorem 10, and $\mathbb{Z}_{n} \leq H \leq S_{n}$

(b) $n=m k, 1<k<n$, $K$ is a nonregular primitive group of degree $p^{m}$, and $H$ is a transitive subgroup of $S_{k}$.

2.1. Proof of Theorem 1. In this section, we deal with the case of $p^{r}=8$ and present a proof of Theorem 1 .

Lemma 13. Let $G$ be a primitive permutation group acting on a set $\Omega$, where $|\Omega|=8$. Assume that $\alpha \in \Omega$. Then, $G$ is one of the following:

$$
\begin{aligned}
& \mathbb{Z}_{2}^{3} \rtimes G_{\alpha} \text {, where } G_{\alpha} \cong \mathbb{Z}_{7}, \Gamma L_{1}(8) \text { or } S L_{3}(2) ; A_{8} ; S_{8} ; \\
& P S L_{2}(7) ; \text { and } P \Gamma L_{2}(7) \text {. }
\end{aligned}
$$

Proof. It is clear that there are no simply primitive groups of degree 8 , so $G$ is doubly transitive on $\Omega$. If $G$ is almost simple, then $\operatorname{soc}(G) \triangleleft G \leq \operatorname{Aut}(G)$. Applying Theorem 6, we find that $\operatorname{soc}(G) \cong \mathrm{A}_{8}$ or $\mathrm{PSL}_{2}(7)$; thus, $G \cong \mathrm{A}_{8}, \mathrm{~S}_{8}, \mathrm{PSL}_{2}(7)$, or $\mathrm{P \Gamma L}_{2}(7)$. If $G$ is affine, then by Hering's result (see [10]), we have $G_{\alpha} \leq \Gamma L_{1}(8)$ which has a cyclic normal subgroup and which is irreducible on $\mathbb{Z}_{2}^{3}$ or $G_{\alpha} \unrhd \mathrm{SL}_{3}(2)$. Since $\Gamma L_{1}(8) \cong$ $\mathbb{Z}_{7}: \mathbb{Z}_{3}$ and $\mathrm{SL}_{3}(2) \cong \mathrm{GL}_{3}(2), G=\mathbb{Z}_{2}^{3}: G_{\alpha}$, where $G_{\alpha} \cong \mathbb{Z}_{7}$, $\Gamma L_{1}(8)$ or $\mathrm{SL}_{3}(2)$.

Now, we prove Theorem 1.

Proof. Let $G$ be a primitive permutation group acting on a set $\Omega$, where $|\Omega|=p^{r}$ for some prime $p$, so $G$ is either doubly primitive or simply primitive on $\Omega$. By Lemma 5 , we know that $G$ is of type HA, AS, or PA. Let $H$ be the socle of $G$.

If $G$ is doubly primitive on $\Omega$, then by Theorem 8 , the type of $G$ is HA or AS, $H \cong \mathbb{Z}_{p}^{r}$ or $T$ where $T$ is nonabelian simple. In the former case, $G=\mathbb{Z}_{p}^{r}: G_{\alpha} \leq \mathrm{A} \mathrm{L}_{r}(p)$ and $G_{\alpha}$ is transitive on the nonzero vectors of $\mathbb{Z}_{p}^{r}$, such subgroups are determined by Hering in [10]. In the latter case, by Theorem 9 , either $G=\mathrm{A}_{p^{r}}$ or $\mathrm{S}_{p^{r}}$ or $\operatorname{PSL}_{n}(q) \leq G \leq \operatorname{P\Gamma L}_{n}(q)$, and $H=A_{p^{r}}$ or $\operatorname{PSL}_{n}(q)$ where $\left(q^{n}-1\right) /(q-1)=p^{r}$. Thus, (a) of Theorem 1 holds.

Assume now that $G$ is simply primitive on $\Omega$. If the type of $G$ is HA, then $G=\mathbb{Z}_{p}^{r}: G_{\alpha} \leq \operatorname{A\Gamma L}_{r}(p) ; G_{\alpha}$ is irreducible but not transitive on the nonzero vectors of $\mathbb{Z}_{p}^{r}$; we obtain (i) (b) of Theorem 1 . If the type of $G$ is AS, then by Corollary 7 , $H \cong \mathrm{PSU}_{4}(2)$ acts simply transitively on $\Omega$ where $|\Omega|=p^{r}=$ 27. Since $H \triangleleft G \leq \operatorname{Aut}(H)$ and $\operatorname{Aut}(H)=\operatorname{PSU}_{4}(2) \cdot 2, G \cong$ $\mathrm{PSU}_{4}(2)$ or $\mathrm{PSU}_{4}(2) \cdot 2$; we have (ii) (b) of Theorem 1. Assume that the type of $G$ is PA; then, $H=\operatorname{soc}(U)^{d} \triangleleft G \leq U \imath K$, where $1<d \leq r, d \mid r$, and $U$ is primitive of degree $p^{r / d}$ of the type AS or SD. Applying Lemma 5, we obtain that the type of $U$ is AS, $\operatorname{son} \operatorname{soc}(U)=T$ is nonabelian, and thus $\operatorname{soc}(U)=$ $T=\mathrm{A}_{p^{r / d}}, \mathrm{PSL}_{n}(q)$ with $\left(q^{n}-1\right) /(q-1)=p^{r / d}, \mathrm{M}_{11}, \mathrm{PSL}_{2}(11)$ with $p=11, r=d, \mathrm{M}_{23}$ with $p=23, r=d$, or $\mathrm{PSU}_{4}(2)$ with $1<d=r / 3$, which are determined by Theorem 6 . Since the wreath product $U>K$ is of type PA, then by Corollary 12 , $K$ is a transitive subgroup of $S_{d}$. Thus, we obtain (iii) (b) of Theorem 1.

This completes the proof of Theorem 1.
The result of Theorem 1 gives rise to the following natural question.

Question 1. Study imprimitive groups of degree $p^{r}$ with $p$ a prime.

\section{Conflict of Interests}

The authors declare that there is no conflict of interests regarding the publication of the paper.

\section{Acknowledgments}

This work is supported by the NNSF (11161058) and YNSF (2011FZ087).

\section{References}

[1] W. Burnside, "On some properties of groups of odd order," Proceedings of the London Mathematical Society, vol. 33, no. 1, pp. 162-184, 1900.

[2] P. J. Cameron, "Finite permutation groups and finite simple groups," Bulletin of the London Mathematical Society, vol. 13, no. 1, pp. 1-22, 1981.

[3] R. M. Guralnick, "Subgroups of prime power index in a simple group," Journal of Algebra, vol. 81, no. 2, pp. 304-311, 1983.

[4] M. W. Liebeck and J. Saxl, “The primitive permutation groups of odd degree," Journal of the London Mathematical Society, vol. 31, no. 2, pp. 250-264, 1985.

[5] C. H. Li and A. Seress, "The primitive permutation groups of squarefree degree," The Bulletin of the London Mathematical Society, vol. 35, no. 5, pp. 635-644, 2003.

[6] C. H. Li, "The primitive permutation groups of certain degrees," Journal of Pure and Applied Algebra, vol. 115, no. 3, pp. 275-287, 1997.

[7] C. E. Praeger, "Note on primitive permutation groups of prime power degree," Journal of the London Mathematical Society (2), vol. 13, no. 1, pp. 191-192, 1976.

[8] M. W. Liebeck, C. E. Praeger, and J. Saxl, “On the O’Nan-Scott theorem for finite primitive permutation groups," Journal of the Australian Mathematical Society Series A, vol. 44, no. 3, pp. 389396, 1988.

[9] E. Dobson and D. Witte, "Transitive permutation groups of prime-squared degree," Journal of Algebraic Combinatorics, vol. 16, no. 1, pp. 43-69, 2002.

[10] C. Hering, "Transitive linear groups and linear groups which contain irreducible subgroups of prime order II," Journal of Algebra, vol. 93, no. 1, pp. 151-164, 1985.

[11] J. D. Dixon and B. Mortimer, Permutation Groups, vol. 163 of Graduate Texts in Mathematics, Springer, New York, NY, USA, 1996.

[12] W. Burnside, Theory of Groups of Finite Order, Cambridge University Press, Cambridge, UK, 1911.

[13] B. Huppert, "Zweifach transitive, auflösbare Permutationsgruppen," Mathematische Zeitschrift, vol. 68, pp. 126-150, 1957. 


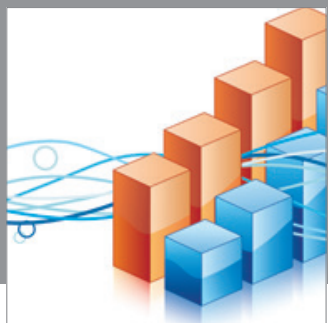

Advances in

Operations Research

mansans

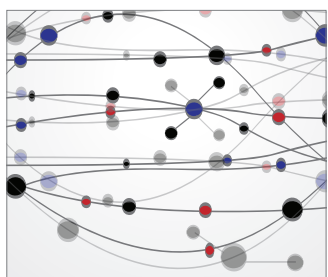

The Scientific World Journal
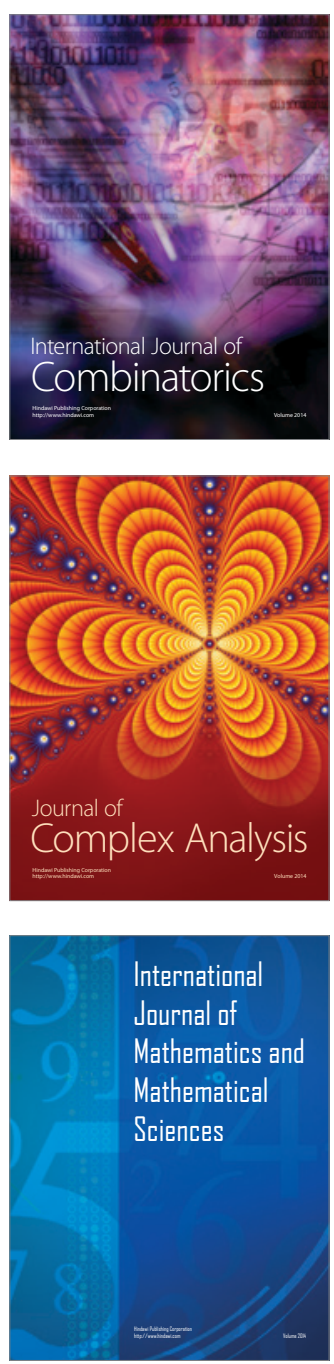
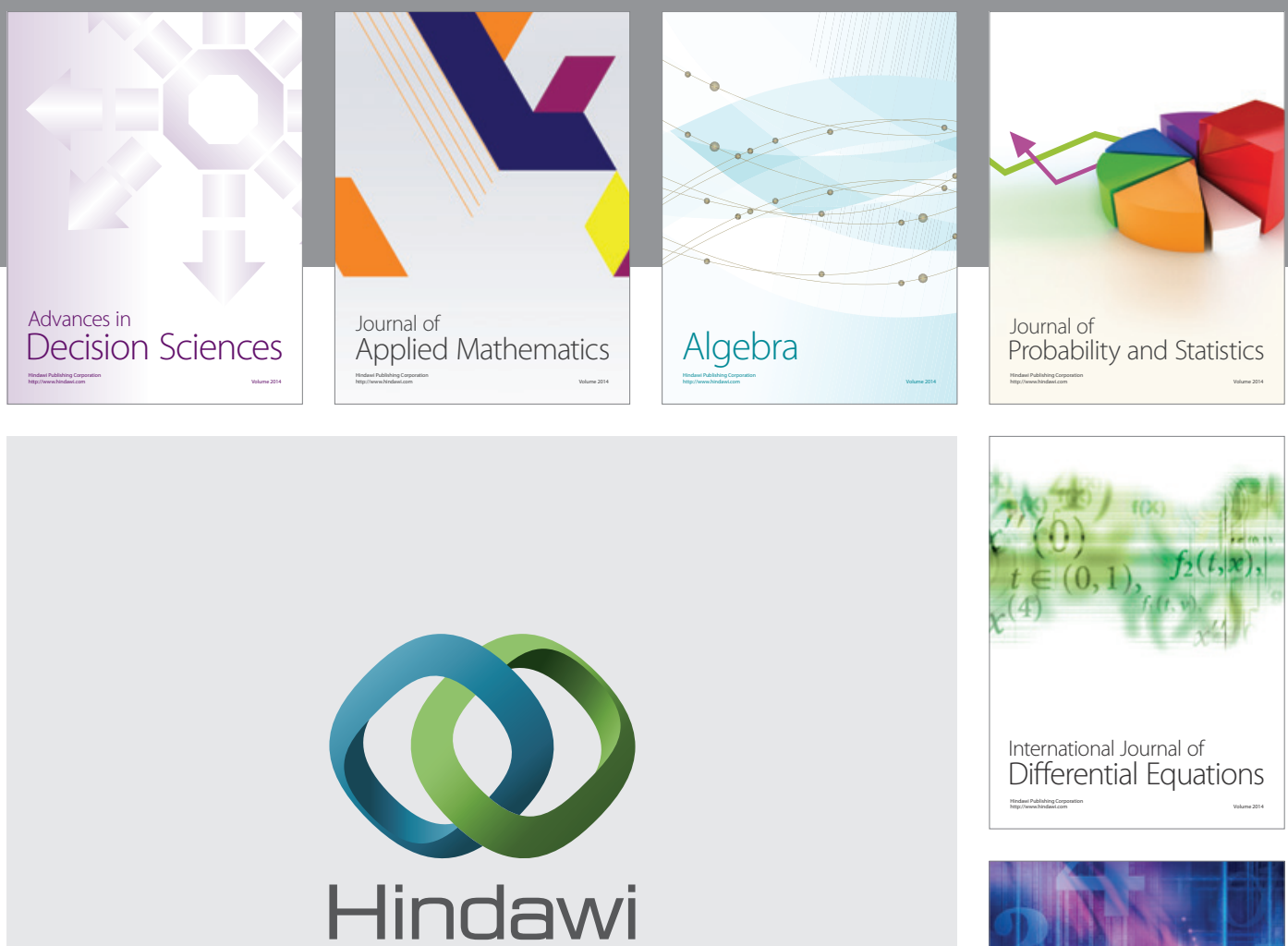

Submit your manuscripts at http://www.hindawi.com
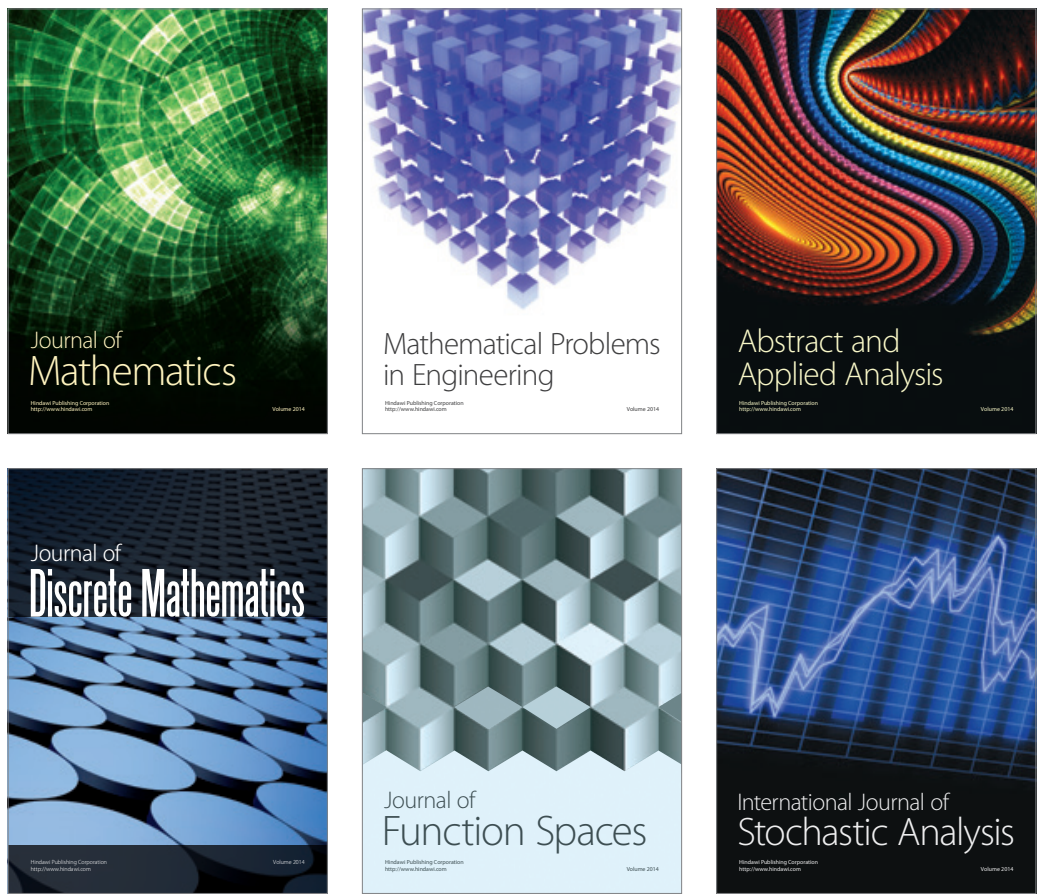

Journal of

Function Spaces

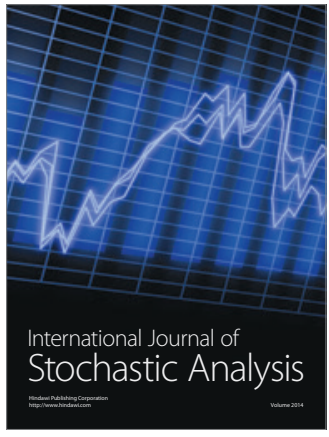

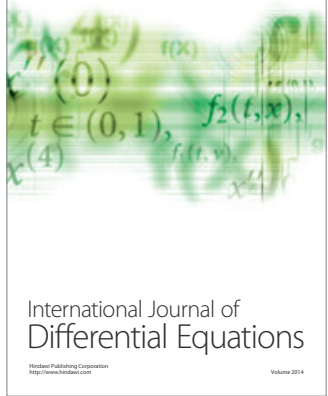
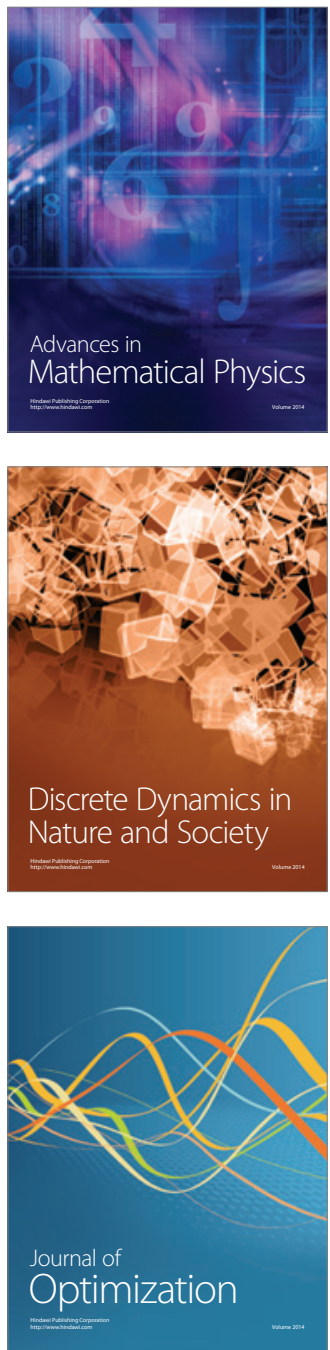